\title{
PIV measurements combined with the motion tracking technique to analyze flow around a moving porous structure
}

\author{
Druault Philippe ${ }^{1,2} \stackrel{\star}{-}$, Germain Grégory ${ }^{3}$, Facq Jean-Valéry ${ }^{3}$
}

${ }^{1}$ University Pierre et Marie Curie-Paris 6, UMR 7190, Institut Jean Le Rond d'Alembert, case 162, 75252 Paris Cedex 5, France

${ }^{2}$ CNRS, UMR 7190, Institut Jean Le Rond d'Alembert, 75252 Paris Cedex 5, France

3 IFREMER, Marine Structures Laboratory, 150 Quai Gambetta, 62321 Boulogne-sur-Mer, France

* Corresponding author : Philippe Druault, email address : philippe.druault@upmc.fr

\begin{abstract}
:
To gain a better understanding of the fluid-structure interaction and especially when dealing with a flow around an arbitrarily moving body, it is essential to develop measurement tools enabling the instantaneous detection of moving deformable interface during the flow measurements. A particularly useful application is the determination of unsteady turbulent flow velocity field around a moving porous fishing net structure which is of great interest for selectivity and also for the numerical code validation which needs a realistic database. To do this, a representative piece of fishing net structure is used to investigate both the Turbulent Boundary Layer (TBL) developing over the horizontal porous moving fishing net structure and the turbulent flow passing through the moving porous structure. For such an investigation, Time Resolved PIV measurements are carried out and combined with a motion tracking technique allowing the measurement of the instantaneous motion of the deformable fishing net during PIV measurements. Once the two-dimensional motion of the porous structure is accessed, PIV velocity measurements are analyzed in connection with the detected motion. Finally, the TBL is characterized and the effect of the structure motion on the volumetric flow rate passing though the moving porous structure is clearly demonstrated.
\end{abstract}

Keywords : Unsteady turbulent flow, Particle Image Velocimetry, Oscillating structure, Fishing structure 


\section{Introduction}

Particle Image Velocimetry (PIV) has become a powerful and elegant measurement method for turbulent flow field. This method has provided a deep insight into complex turbulent flows especially those around moving interfaces where fluid-structure interaction problems (Jeon and Sung, 2011 and Druault et al., 2012) or two-phase flows (Khalitov and Longmire, 2002) are encountered. These last flow configurations are of fundamental importance in many engineering fields like fluid machinery, insect flying, and fish swimming. However, one of the great difficulties for PIV measurements around an arbitrarily moving body and/or in the presence of two-phase flows is that of determining the position of the moving deformable interface, especially when the interface is not clearly visible. Another great related difficulty remains the measurements of the flow velocity around the interface. 
When dealing with planar PIV measurement techniques, a lot of previous studies have focused on the development of algorithmic masks (see [1, 4] and references therein). But when dealing with flow around a porous fishing structure, the implementation of specific post-processing tools aimed to detect the deformable porous interface remains quite complex. A solution may consist in treating each PIV frame manually [2] but such a solution is not satisfactory especially when dealing with numerous PIV snapshots. Moreover, in certain cases, the fishing net interface is not clearly visible (see §2.2). The main issues are related i) to the turbulent flow that develops not only on the upper part of the fishing net but also on the lower part of the net and ii) to the fact that the fishing net structure is porous and so allows the fluid to pass through. Moreover, the fishing net structure is composed of different net panels which are quite different from each other (incidence, porosity, ...). For instance, hydrodynamic forces acting upon the horizontal part of the fishing net structure undergo relatively small amplitude oscillations at low frequencies. Conversely, a turbulent flow passing through inclined porous structures may induce stronger oscillations and the wake flow developing behind the cod-end net structure induces also large cod-end oscillations [5].

Due to such difficulties, very few studies have been performed to elucidate the flow developing over such porous structures. Nevertheless, the determining of the turbulent flow is of great interest for a better understanding of selectivity (that is the ability of fishing gears to prevent catches of nontarget fish) and also for the development of numerical codes enabling the determining of the fishing gear drag force [6]. In this last case, the knowledge of flow velocities as well as the frequencies structure oscillations will improve notably numerical modelling. For instance, knowing that the frequency body oscillations may have an effect on the drag of the oscillating body [7,8], it will be interesting to access the effect of the oscillations on the modification of the local fluid velocity and then ultimately on the resulting drag force.

In short, previous investigations have generally been limited to a simple geometry rigid cod-end structure [5,9] or stiff net panels (grids) [10]. Kim [11] performed one-point measurements inside a realistic cod-end of a shrimp beam trawl. It was confirmed the importance of turbulence affecting the maneuverability of fish swimming. Very recently, Time-Resolved Particle Image Velocimetry (TRPIV) measurements have been carried out to investigate the Turbulent Boundary Layer (TBL) flow developing over the horizontal part of a fishing trawl model [2]. In this last study, the determining of the motion of the fishing net structure during PIV measurements was done manually and can not be reproduced for a full investigation of the whole flow field all around a fishing net structure. On the other hand, previous studies have also focused on the analysis of the hydrodynamics around sea fish cages $[12,13]$. This is of great interest for the future development of such cages due to the great impact of hydrodynamics on fish health and escape [13]. It was then observed that when exposed to current, a fish cage undergoes deformation which leads to the modification of the fish environment. This is directly related to hydrodynamics and associated parameters such as inclinations of structure, flow speed, porosity of the structure, ... [14].

In this paper, we propose an advanced experimental methodology enabling the post-processing of PIV images to determine the fishing structure interface and the measurements of velocities. This methodology relies on an efficient combination of a procedure aimed at tracking the local motion and deformation of the interface and also the TRPIV measurements. A representative piece of fishing net structure is considered allowing the investigation of both the Turbulent Boundary Layer (TBL) developing over horizontal porous moving fishing net structure and the turbulent flow passing through the moving porous inclined structure. The two-dimensional velocity vectors are measured around this oscillating structure and the motion of this structure is simultaneously measured with a motion tracking technique. Some interactions between the turbulent flow development and the fishing net structure oscillations are analyzed. 

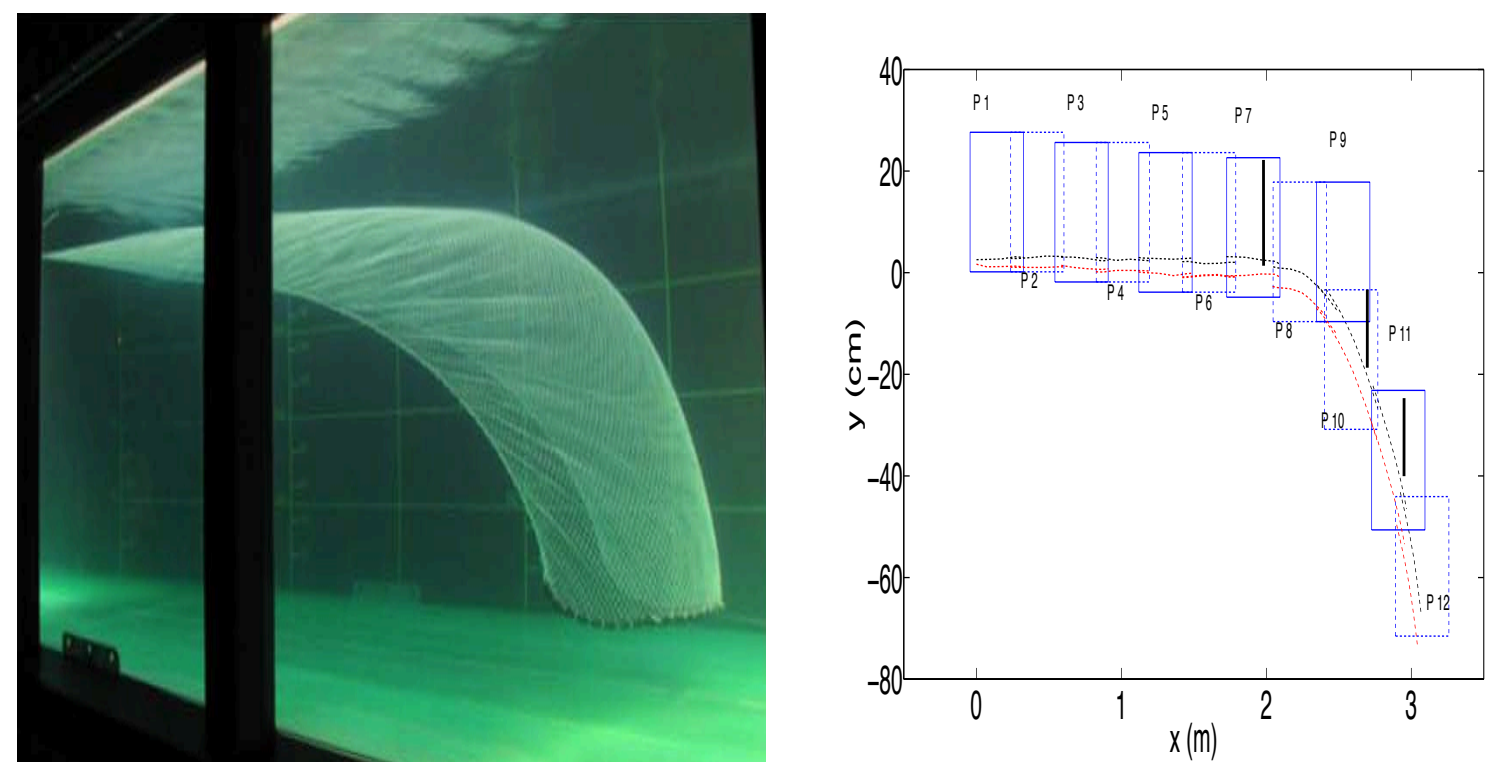

Figure 1: Left hand side: Fishing net structure under investigation. Right hand side: Overlapped PIV measurement planes located in the median plane of the porous structure. Red and black dotted lines indicate the lowest and highest position of the structure during measurements, respectively. Black vertical lines (in planes 7, 10 and 11) indicate the lines along which velocity spectra as well as volumetric flow rates are computed in the following sections (see $\S 3.2$ and 3.4).

\section{Materials and methods}

\subsection{Experimental device}

Tests were performed at the IFREMER (French Research Institute for Exploration and Exploitation of the Sea) wave and current flume tank. The flume tank working section is $18 \mathrm{~m}$ long by $4 \mathrm{~m}$ wide and $2 \mathrm{~m}$ deep. The flow turbulence in the tank is $3 \%$ and can be increased to $15 \%$ when removing the flow straighteners. The streamwise flow velocity range is 0.1 to $2.2 \mathrm{~m} \cdot \mathrm{s}^{-1}$. More details about the flume tank can be found in $[5,9]$.

In the following, measurements are carried with a fixed input streamwise velocity of $U_{r e f}=0.8 \mathrm{~m} . \mathrm{s}^{-1}$ for which the turbulence intensity is less than $3 \%$.

In this study, a $2 \times 3 \mathrm{~m}^{2}$ rectangular sheet of net ( 80 meshes width by 120 meshes length), held in place in the flow by 2 ropes, is used (see figure 1-left hand side). The sheet of net is made of polyamide twine of $2.2 \mathrm{~mm}$ diameter and diamond-shaped meshes of $22 \mathrm{~mm}$. This piece of fishing net structure is positioned at the middle of the testing section, where no wall effects are encountered.

This simplified structure is chosen because of the flow and the shape taken by such a flexible porous structure. Two parts can be easily identified: a horizontal one enabling the development of a TBL flow and an inclined part (from 0 to 62 degrees) enabling the flow to pass through the open meshes. These parts can be seen on classical fishing devices and mainly on pelagic and bottom trawls $[6,10]$. Furthermore, the presence of a free downstream end which is the main cause of structure oscillations can be related to aquaculture fishing cages. In such a case, similar hydrodynamic behaviour can be observed and the analysis of flow passing through sea fish cages is also of great interest due to its connection with fish health (see [13] and references therein).

\section{$2.2 \quad$ Flow measurements}

The motion tracking technique and PIV measurement technique are successively described. 


\section{Motion tracking technique}

In order to characterize the motion of the structure, a motion tracking system is used and applied directly to raw PIV images presented below. In this aim, targets made of $8 \mathrm{~mm}$ diameter floats are fixed each 4 knots of the net in the flow direction. The targets are not in the main symmetrical axis of the piece of net in order to not disturb the flow measurement at the same time. The introduced gap inserted is more or less half a length mesh (approximately $2 \mathrm{~cm}$ ). As it has been observed by Tomkins and Adrian [15], the three dimensional coherent structures present in turbulent boundary layer flow over a smooth, flat plate are very elongated close to the wall, with a small spanwise extent. Moreover, an estimation of the current boundary layer thickness indicates that it never exceeds $8 \mathrm{~cm}$ in the vicinity of the net structure (see figure 14). Consequently, we think that this gap is sufficient to be sure that the balls do not perturb the flow in the measurement plane.

Ma-studio software [16] with a precision less than $1 \mathrm{~mm}$ is used to track the target in each PIV image. The algorithm relies on the pattern matching approach detailed in [17]. Briefly, it consists of determining manually the initial positions of the four targets in the first PIV image. Then, the Pattern Matching algorithm scans the other PIV images allowing the recognition of area (target) that matches best the initial pattern. The motion of the target surface is found to be negligible for any image pair because of the short time separating the double frame PIV images.

\section{Particle Image Velocimetry measurements}

The velocity measurements are made by means of a 2D PIV system. The PIV apparatus is based on a two-chamber Gemini Nd-Yag laser $(2 \times 120 \mathrm{~mJ} /$ pulse at $15 \mathrm{~Hz})$, using an excitation wavelength of $532 \mathrm{~nm}$. The laser sheet is emitted in the water, in the vertical plane centered along the main axis of the piece of net. The thickness of the laser sheet is $2 \mathrm{~mm}$ in all the measurement planes, so that there is no refraction and no disturbance induced by the floats used by the motion tracking system. The camera (Hi-sense CCD camera) recording PIV images has a $4 \mathrm{~Hz}$ frequency for double-frame images with a $1280 \times 1024$ pixels $^{2}$ resolution. The distance between the camera and the laser sheet is $2.2 \mathrm{~m}$ so that the image size is $40 \times 30 \mathrm{~cm}^{2}$ (the camera is placed perpendicular to the laser sheet). The instantaneous velocity fields are obtained using a cross-correlation PIV algorithm. The size of the interrogation window is $16 \times 16$ pixels $^{2}$ and adjacent windows are $50 \%$ overlapped. That leads to obtain $\left(n_{x} \times n_{y}\right)=(199 \times 149)$ points with a similar grid spacing of $1.9 \mathrm{~mm}$ in each direction. The frequency resolution is sufficient (cutoff frequency of $2 \mathrm{~Hz}$ ) to investigate the effect of low frequency body oscillations (less than $1 \mathrm{~Hz}$ ) on velocity measurements.

Figure 1 (right hand side) represents the whole set of 2D PIV measurement planes located in the vertical median plane of the porous structure. In this vertical plane, no edges effects may be observed [18]. In each plane, 818 instantaneous velocity vectors are measured. Streamwise $(x-$ direction) and transverse ( $y$-direction) velocity components are then available on the regular $2 \mathrm{D}$ mesh grid. Thanks to the determining of fishing structure position, automatic masks are generated and applied to the PIV planes.

Figure 2 displays two instantaneous raw PIV images obtained in two different measurement planes. We observe that the porous surface of the structure may not be easily identifiable in raw PIV images. That emphasizes the use of another measurement technique enabling the tracking of the random motion of the porous structure during PIV measurements.

\section{Results}

In this study, we combine the structure motion capture and PIV measurements in order to establish a link between the motion of a flexible porous structure and the flow characteristics governing its dynamic.

The 2D motion of the moving structure is first analyzed. Then, the frequency content of selected PIV velocity vector fields is investigated and linked to the body frequency oscillations. The TBL flow and its associated classical thicknesses are then regarded thanks to an analysis in the structure frame of reference. Finally, the flow passing though the inclined moving structure is studied as a 

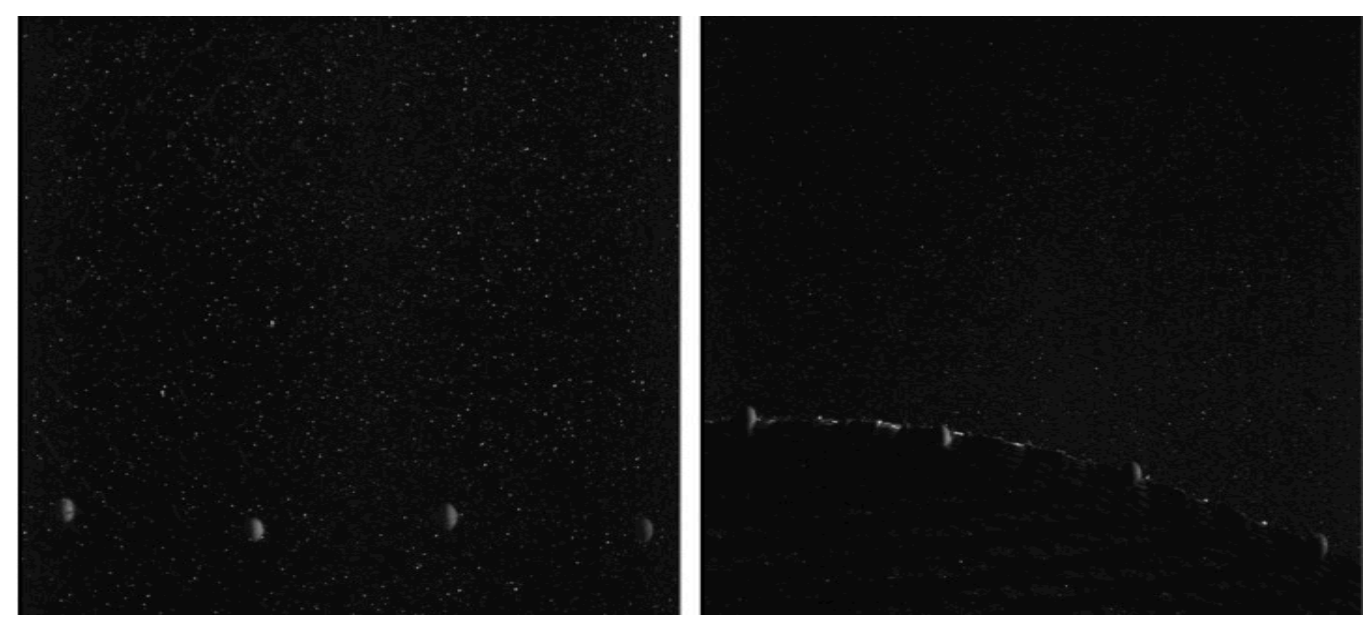

Figure 2: Selected instantaneous raw PIV images obtained in plane 2 (left hand side) and in plane 8 (right hand side). In these graphs, the floats represent targets allowing the instantaneous characterization of the moving deformable interface.

function of body oscillations.

\subsection{Analysis of the motion of the porous structure}

As a first step, the transverse $(y-)$ as well as the streamwise $x$-oscillations of the structure in each measurement plane are analyzed thanks to the motion tracking measurements. Notice that the motion of the porous structure can be more or less assimilated as a rigid body structure because each of the four targets located in a same measurement plane follows a quasi-identical 2D motion. Thus, in the following, the motion of one target is only considered for the 2D body oscillation characterization. As an illustration, figure 3 represents the time evolution of the fluctuating transverse and streamwise motions (the mean values have been subtracted) of the body during selected PIV measurements (planes 2, 6 and 11 given in figure 1). In the graph, the time evolutions of body oscillations are superimposed in a similar scale but are translated along the vertical axis to better appreciate the amplitudes of the associated oscillations. The standard deviations (Root Mean Square-RMS) of structure oscillations in each individual PIV plane are also plotted in figure 4. We observe at first that the amplitudes of $x$-oscillations are very small compared to the $y$-oscillation ones. More precisely, when comparing the peak amplitude of both $x$ and $y$ fluctuating displacements given in figure 3 and also the peak RMS values (figure 4), a ratio of 3 is obtained for measurement planes 2 and 11 and a ratio of 12 for measurement plane 6 . Moreover, the $x$-oscillations are mainly present at the beginning and at the end of the porous structure. Indeed, the maximal values of the RMS are obtained at the beginning and at the end of the structure. Conversely, the amplitude of $y$-oscillations increases as a function of $x$ axis, apart from around $x \approx 2$ that corresponds to the beginning of the inclined part.

In each measurement plane, one also computes the time average correlation coefficient $R_{x y}$ between $x$ - and $y$-displacement signals. Table 1 provides $R_{x y}$ values. It is noticeable to observe that near the end of the fishing structure, both time oscillation signals are quite very well correlated. Indeed, it exists a strong coupling between $x$ - and $y$-oscillations, especially along the inclined part. This coupling results from the flow interaction with the chain located at the end of the fishing structure (see figure 1-left hand side). This chain used to maintain the porous structure in incidence to the uniform flow is simultaneously subject to $x-$ and $y$-oscillations. When regarding the displacement of the horizontal parts (measurement planes from 1 to 7 ), no real coupling exists between streamwise and transverse structure oscillations. The viscous boundary layer flow developing on both side of the 


\begin{tabular}{|c|c|c|c|c|c|c|c||c|c|c|c|c|}
\hline $\begin{array}{c}\text { Plane } \\
\text { number }\end{array}$ & 1 & 2 & 3 & 4 & 5 & 6 & 7 & 8 & 9 & 10 & 11 & 12 \\
\hline$R_{x y}$ & 0.16 & 0.17 & 0.25 & 0.23 & 0.22 & 0.38 & 0.50 & 0.60 & 0.64 & 0.74 & 0.75 & 0.91 \\
\hline
\end{tabular}

Table 1: Correlation coefficient computed between $x$ - and $y$-displacement signals obtained in each individually measurement plane. Horizontal part: Planes from 1 to 7 . Inclined part: Planes from 8 to 12 .

net structure [19] exerts a shear stress on the porous fluttering structure that affects both streamwise and transverse structure motions in a different manner.

To investigate further the frequency content of $x$ - and $y$-oscillations, the associated frequency spectra are computed. Recall that the sampling frequency of PIV measurements is $4 \mathrm{~Hz}$ and the time duration corresponds to $204.5 \mathrm{~s}$. Then when computing FFT, the frequency domain is limited to $[0: 2] \mathrm{Hz}$ and the frequency resolution is $0.0049 \mathrm{~Hz}$. We have in mind that such limited time duration places some constraints on data analysis. However, for present spectral analysis, the frequency domain as well as the frequency resolution seem to be sufficient to properly estimate the frequency content of available signals. Indeed, these signals are of very low frequency content. Furthermore, note that to enhance the statistical convergency, present spectral analysis are performed using FFT based on Welchs method and FFT is computed based on three blocks of 512 samples with $50 \%$ overlapping and a Hann window is applied to each block. In such a case, the frequency resolution is $0.0078 \mathrm{~Hz}$ which seems also sufficient for present spectral analysis. Furthermore, about the computation of the Power Spectral Densities as well as coherencies presented in the following figures, we do not observe significant differences especially in the low frequency domain when using different sizes of block superior to 512 samples. That permits to state about the consistency of the observed low frequency content of available signals. Figure 5 (top) shows spectra of both oscillations in two PIV planes (horizontal part, plane 2 and inclined part, plane 12). The spectra are normalized with the local RMS value. In Fig. 5 (top-left), the spectra are plotted up to the Nyquist frequency $(2 \mathrm{~Hz})$ demonstrating that no-aliasing problems are present. Moreover, to better appreciate the low frequency content of the available signals, a zoom view ( $x$-axis is limited to $0.5 \mathrm{~Hz}$ ) of these spectra is also given in Fig. 5 (top-right). The spectra obtained in plane 12 exhibit a dominant low frequency component and also some frequency peak around $f=0.2 \mathrm{~Hz}$.

This figure also displays iso-surfaces of the spectra as a function of $x$-locations. Indeed, in each available PIV plane, one computes the FFT of body $x$-oscillations as well as of body $y$-oscillations. In each spectrum graph, the spectra are normalized with the local RMS value. At the end of the structure, $x-$ and $y$-oscillations exhibit mainly a low frequency component smaller than $0.1 \mathrm{~Hz}$. These frequencies are also recovered for the $x$-oscillations at the beginning of the porous structure, $x<0.5 \mathrm{~m}$. As stated above, this low frequency component is linked to the flow interaction with the net structure which is also coupled to the elastic character of the net structure. Note that previous studies based on the measurements of cable force allowing to maintain present fishing net structure horizontally have shown that the cable force signal is also of low frequency content [20]. A mid frequency component is retrieved at the end of the horizontal part $(x \approx 1.5-2 \mathrm{~m})$ for both streamwise and transverse oscillations. In this last zone, the turbulent flow greatly interacts with the net structure and this area may be also viewed as the beginning of the large scale wake flow developing behind the whole net structure. When regarding $y$-displacement spectra, no main frequency peak is observed along the horizontal part of the fishing structure (until measurement plane 6).

To analyze the couplings between $x$ - and $y$-displacement signals at the end of the porous structure and the ones observed along the horizontal part, the coherence function $C$ defined as follows:

$$
C_{x_{1} x_{2}}(f)=\frac{\left|G_{x_{1} x_{2}}(f)\right|}{G_{x_{1} x_{1}}(f) G_{x_{2} x_{2}}(f)}
$$

where $G_{x_{1} x_{2}}$ is the cross-spectral density between $x_{1}$ and $x_{2}$ signals. This coherence function allows the calculation of the common frequency between two signals located 1) on the horizontal part $\left(x_{1}\right.$ 

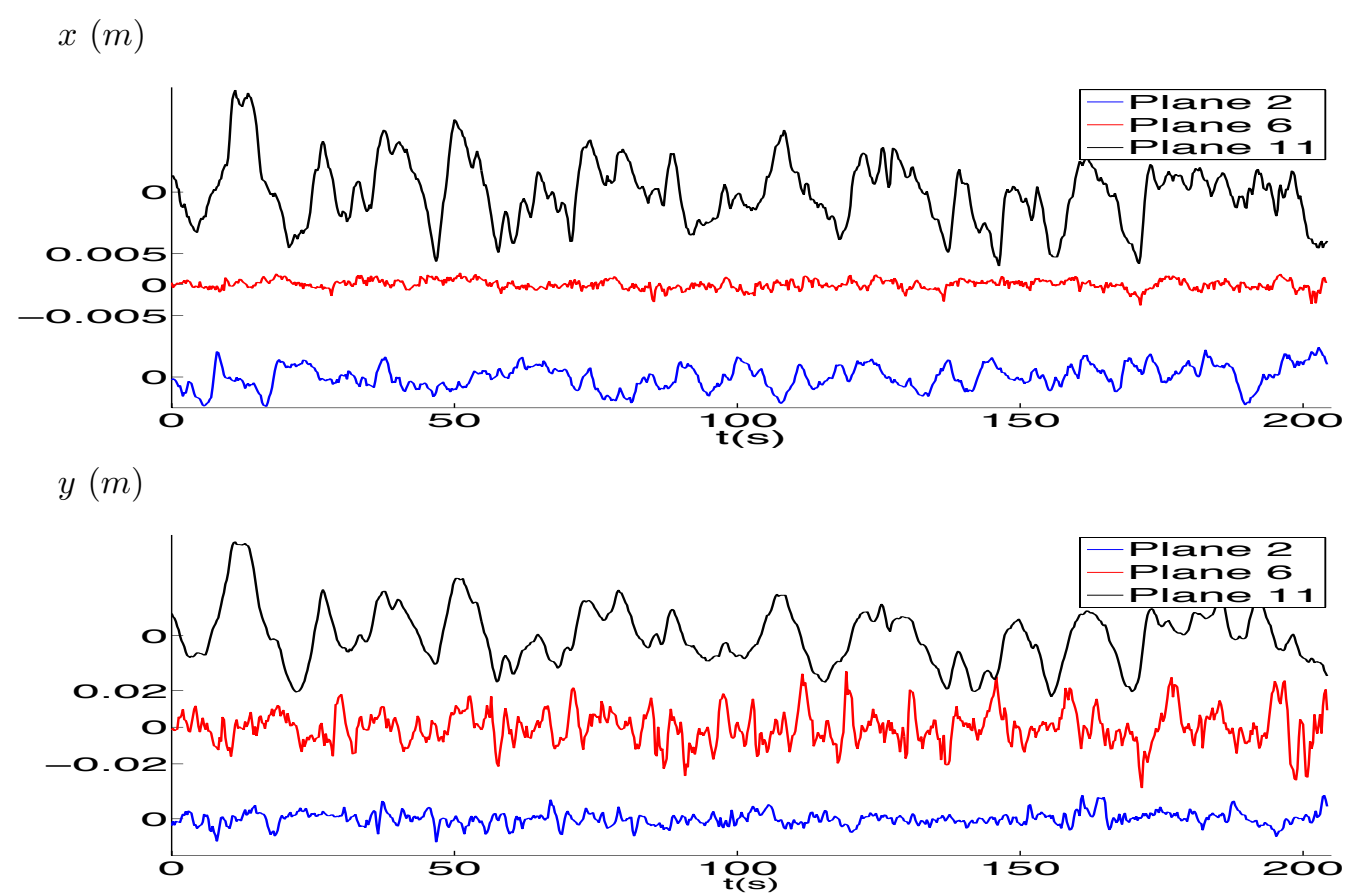

Figure 3: Time evolution of the longitudinal (top) and transverse (bottom) motion of the porous structure during PIV measurements in 3 planes (2,6 and 11). In these figures, the fluctuating part of the motion is presented in a similar scale but translated along the axis to understand the amplitude of the associated fluctuations more easily.

then corresponds to either $x$ - or $y$ - oscillation signals) and 2) at the end of the net structure $\left(x_{2}\right.$ then corresponds to $x$ - or $y$-displacement signals). Figure 6 represents the coherence between $x$-displacement signal obtained in planes 2 and the one in plane 12 . It is also displayed the coherence between both $y$-displacement signals obtained in these planes. It is then observed that both $x$-displacement signals as well as both $y$-displacement signals are well correlated at selected very low frequencies. Globally, the levels of $x$-displacement coherence are slightly superior to those of $y$-displacement coherence and a quite reasonable coherence $(\approx 0.5)$ is obtained in the frequency domain under interest.

Globally, such a frequency oscillation analysis provides interesting features about the fluttering of the net structure under the effect of turbulence. This may have some consequences on fish escape. Indeed, Kim and Whang [21] have shown recently the effect of turbulence on net panel fluttering which acts as an active stimulating device for increasing the fish escape. Moreover, high amplitude $y$-oscillations observed at the free downstream end have important consequences on hydrodynamics passing through the structure. Thus, assuming that present hydrodynamics are similar to the ones developing around the tapered end of commercial fish cages, such high amplitude oscillations may induce important potential modifications of fish cages (deformation, porosity, fish behavior, ....) [13].

\subsection{Spectral analysis of PIV velocity measurements}

In this section, two specific measurement planes (one along the horizontal part and the other one along the inclined part) are used to study the spectral content of the instantaneous velocity fields. More precisely, the flow developing around the moving structure is considered at a specific $x$ location in plane 7 (TBL flow) and in plane 11 (flow passing through the structure) respectively. For a fixed $y$-line domain always located above the moving structure (see figure 1 where black vertical lines 


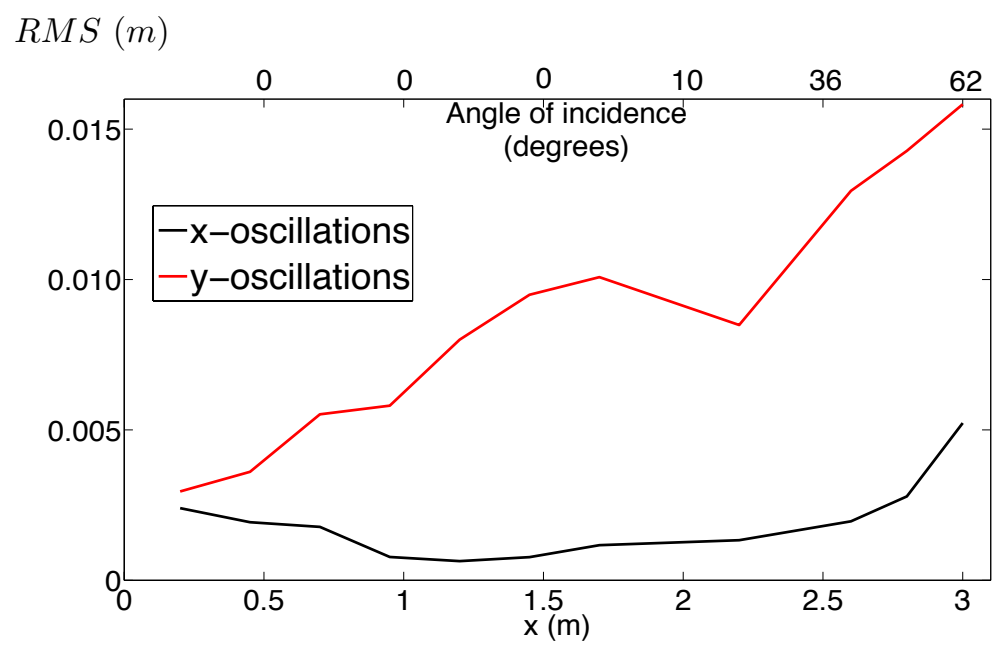

Figure 4: Root Mean Square of the streamwise (black line) and transverse (red line) motion of the structure as a function of the $x$-locations. The upper horizontal axis provides associated values of angle of incidence along the $x$-axis.

indicate the $y$-line under investigation in each PIV plane), we save instantaneous transverse and streamwise velocity components.

Figures 7 and 8 represent velocity spectra represented as a function of $y$-positions ( $x$-axis) and frequencies $(y$-axis), for the TBL flow and for flow passing through the structure, respectively.

Concerning the TBL flow (figure 7), we observe that the transverse velocity component is very sensitive to the porous structure oscillations. Indeed, the transverse velocity spectrum exhibits a dominant low frequency component which is similar to the one deduced from 2D body oscillations. Conversely, the streamwise component is more associated with the turbulent flow structure passage. Thus, the associated spectrum exhibits a higher frequency content. These differences can be related to the flow passing through the horizontal part of the structure during its oscillations. Indeed, the transverse velocity component is more affected by $y$-oscillations of the structure allowing the passage of fluid in this direction. Otherwise, a frequency peak around $0.24 \mathrm{~Hz}$ is obtained for both velocity components. It may be associated with a specific large scale vortex organization and/or flow interaction with the transverse flow passing through the porous structure.

Concerning the flow passing through the structure (figure 8), a low frequency component is obtained for the spectrum of the streamwise velocity component. This can be associated with $y$ oscillations of the porous structure. Such a component is very sensitive to the structure oscillations as will be confirmed in the following section regarding the effect of structure oscillations on the volumetric flow rate. Conversely, the transverse velocity spectrum exhibits a broader spectrum.

To confirm these couplings between the structure oscillations and the flow, the coherence function (equation 1) is computed between instantaneous velocity component extracted along the fixed $y$-line and $x$ - or $y$-oscillations. Figures 9 and 10 display such coherence function determined in plane 7 and plane 11 respectively. It is then observed that in both planes, the transverse velocity component does not exhibit marked common frequencies. Some common frequencies around $f \approx 0.2 \mathrm{~Hz}$ have just been observed in the far field of the boundary layer flow (Plane 7). As stated previously, this can be related to the beginning of the large scale vortex sheet developing behind the fishing structure. Conversely, the coherence function computed between the streamwise velocity component and displacement signals shows that a great correlation is observed at very low frequency $(f<0.1 \mathrm{~Hz})$ in plane 11. Then, there exist a strong coupling between low frequency oscillations and streamwise velocity component. Moreover, in plane 7 , an high coherence value is mainly observed in the vicinity of the TBL flow and for frequency inferior to $0.3 \mathrm{~Hz}$, especially for the coherence function computed between the streamwise velocity component and $y$-oscillations. 


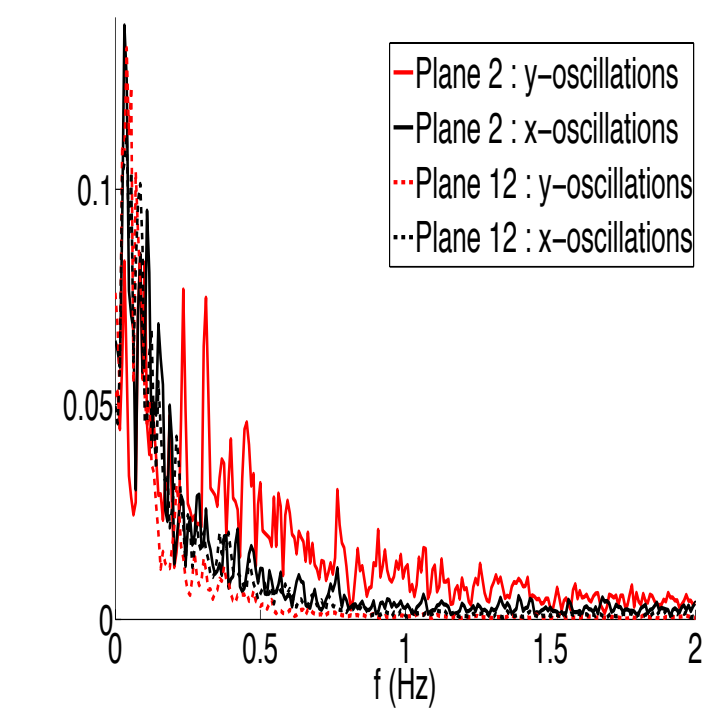

$\mathrm{f}(\mathrm{Hz})$

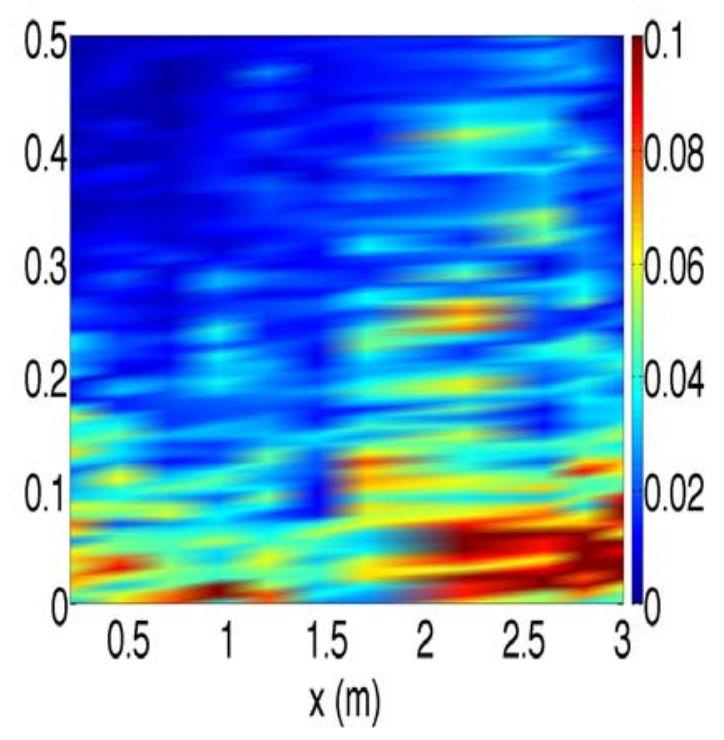

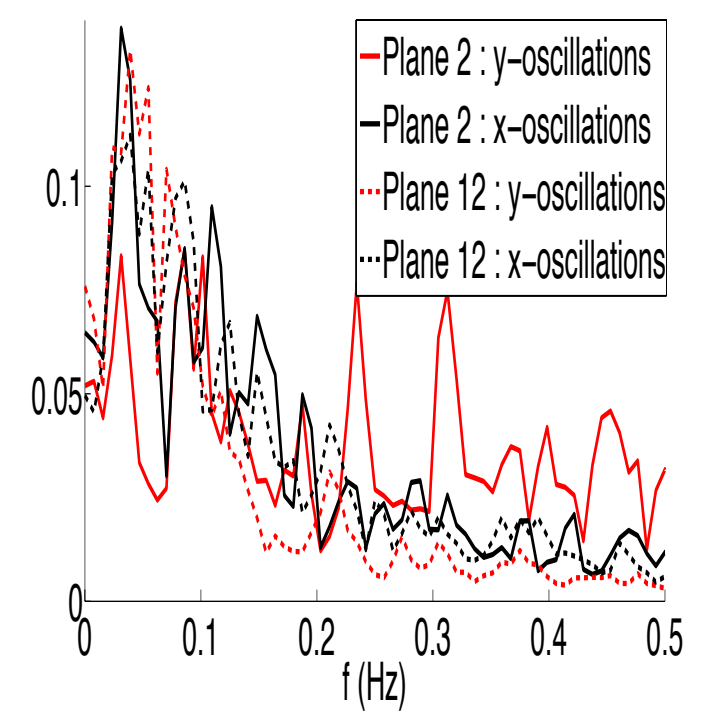

f $(\mathrm{Hz})$

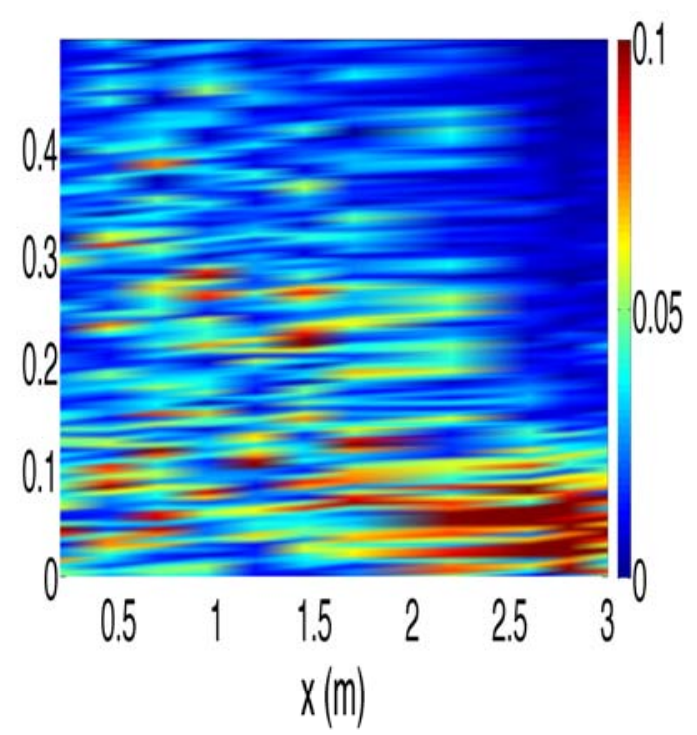

Figure 5: Normalized frequency spectrum of the transverse and longitudinal motions of the fishing net structure during PIV measurements in planes 2 and 12, as a function of frequency up to Nyquist frequency (Top-left) and a zoom view for frequency in $[0: 0.5] \mathrm{Hz}$ (Top-right). Bottom (left): normalized frequency spectrum of the structure $x$-oscillations as a function of streamwise positions. Bottom (right): normalized frequency spectrum of the body $y$-oscillations as a function of streamwise positions. 

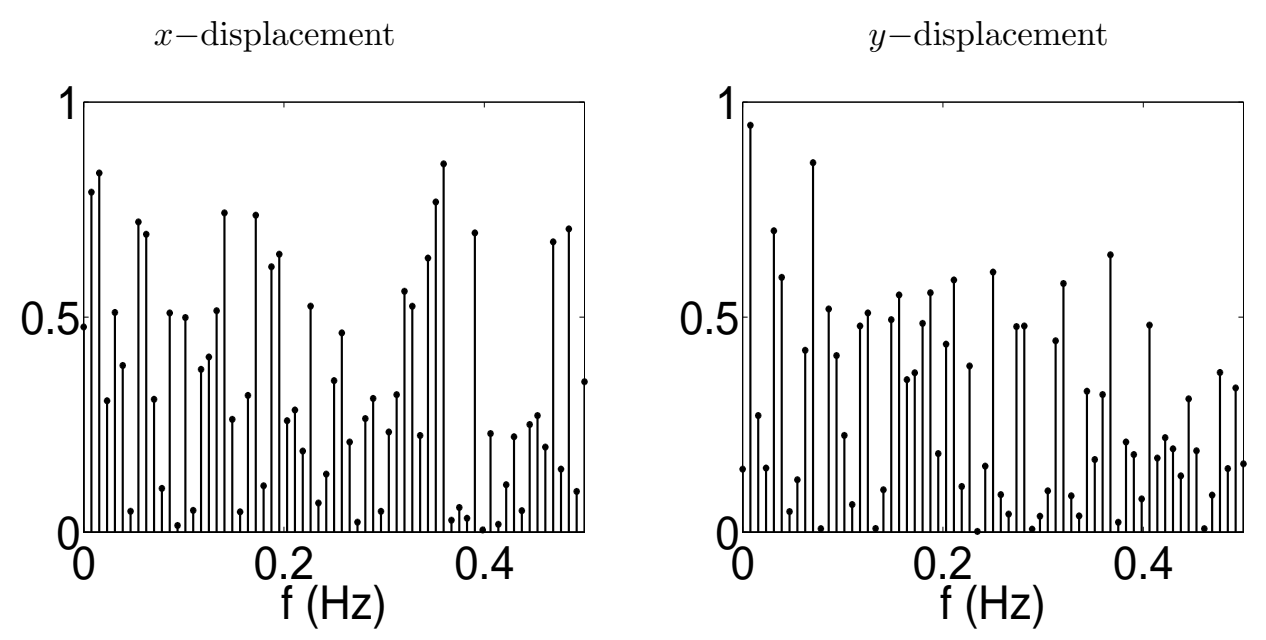

Figure 6: Left hand side: Coherence function computed from the $x$-displacement signals of Planes 2 and 12. Right hand side: Coherence function computed from the $y$-displacement signals of Planes 2 and 12 .

\section{Synthesis}

In the present flow configuration, three main flow interactions are present : i) the turbulent boundary layer which interacts with the horizontal part of the fishing structure, ii) the flow which passes through the structure and iii) the flow which interacts with the chain located at the end. The complexity of these flow interactions is reinforced with the fact that the net structure is elastic and free to move.

On the one hand, the low frequency component of structure oscillations is quite similar to some flap case involving low frequency. But presently, the end of the structure which is composed of a chain allowing to maintain the net structure in incidence with the flow, is slightly different to classical flap case.

On the other hand, a wake flow is developed behind the net structure. Taking into account the fluttering motion of the structure, its transverse displacement extent is approximately $l=0.80 \mathrm{~m}$ (see figure 1 right hand side). Then based on conventional theory, the vortex shedding street developing behind a structure body corresponds to a Strouhal number of $S t=U_{r e f} \times f / l$ approaching 0.2 . That leads to a vortex shedding frequency of $0.21 \mathrm{~Hz}$. In this sense, frequencies detected around $0.2 \mathrm{~Hz}$ (Plane 7 for instance) may be related to such vortex shedding street.

One of the great difficulty for the analysis of flow mechanisms associated with each marked frequency concerns the numerous length scales of the flow which are directly related to the dimensions of the net fishing structure. Recall that it consists of a rectangular sheet of net $\left(3 \times 2 \mathrm{~m}^{2}\right)$ which is made of polyamide twine of $2.2 \mathrm{~mm}$ diameter and diamond-shaped meshes of $22 \mathrm{~mm}$. Besides, each part of the net structure is subjected to displacements modifying the local mesh opening.

Finally, previous results emphasize the great complexity of present flow interaction but they underline the couplings existing between structure displacements and hydrodynamics. In the following, further analyses will be performed in this sense.

\subsection{Mean boundary layer flow characteristics}

Based on available measurements, the mean characteristics of the TBL flow can be accessed by working within the reference frame of the porous structure as in [22]. In the following, the TBL mean flow field is investigated leading to the analysis of the classical TBL thicknesses. 

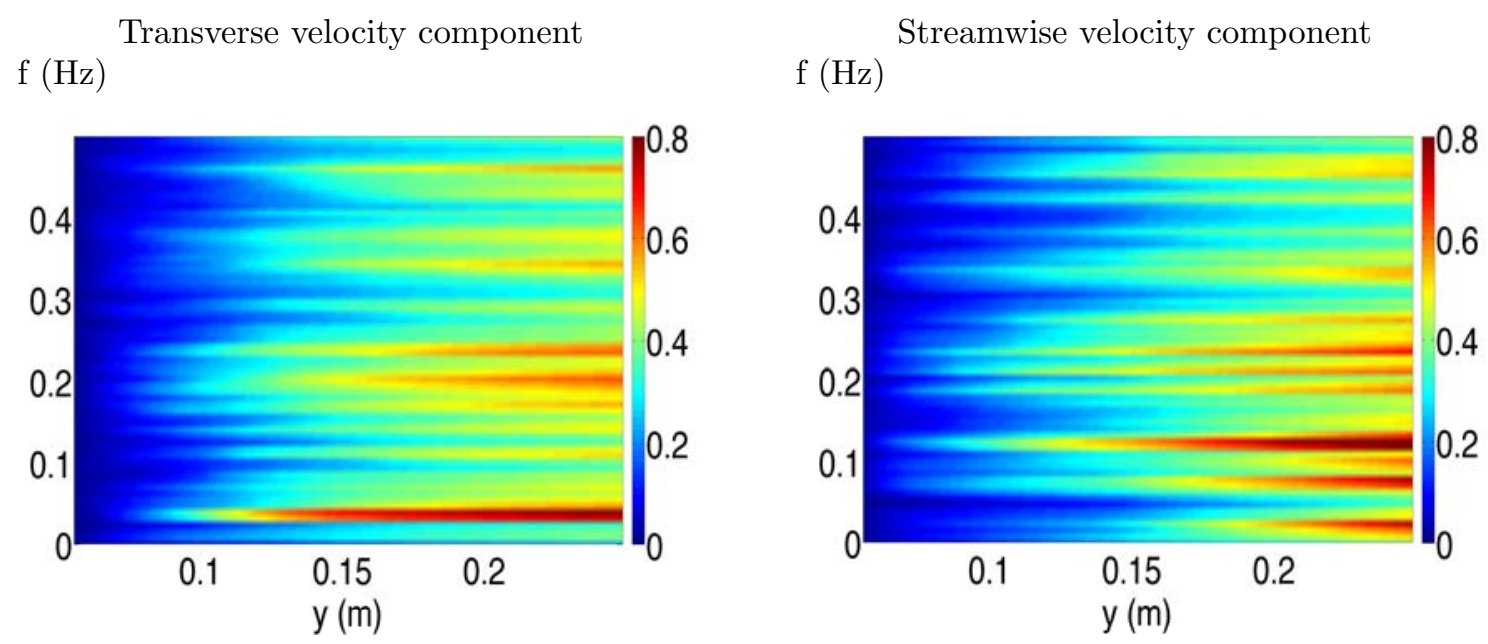

Transverse component spectrum

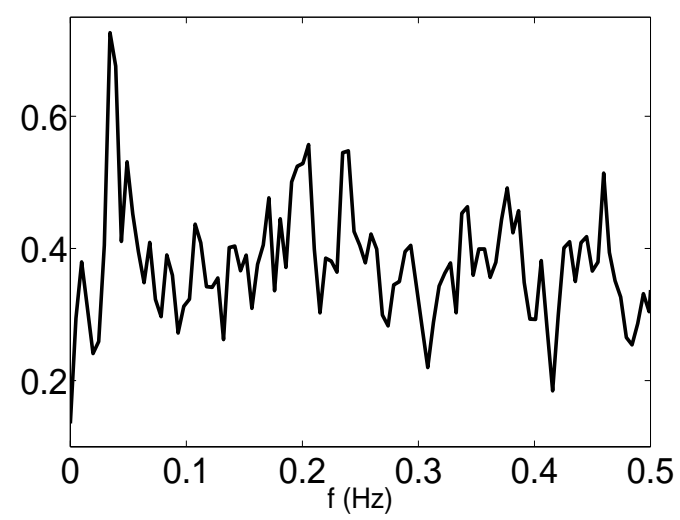

Figure 7: Boundary layer flow (Plane 7). Normalized frequency spectrum of the transverse (top-left) and streamwise (top-right) velocity components along the line $x \approx 2 \mathrm{~m}$ and $y>0.05 \mathrm{~m}$. Bottom: Normalized frequency spectrum at the point $(x, y)=(2,0.17)$ for the transverse velocity component. 

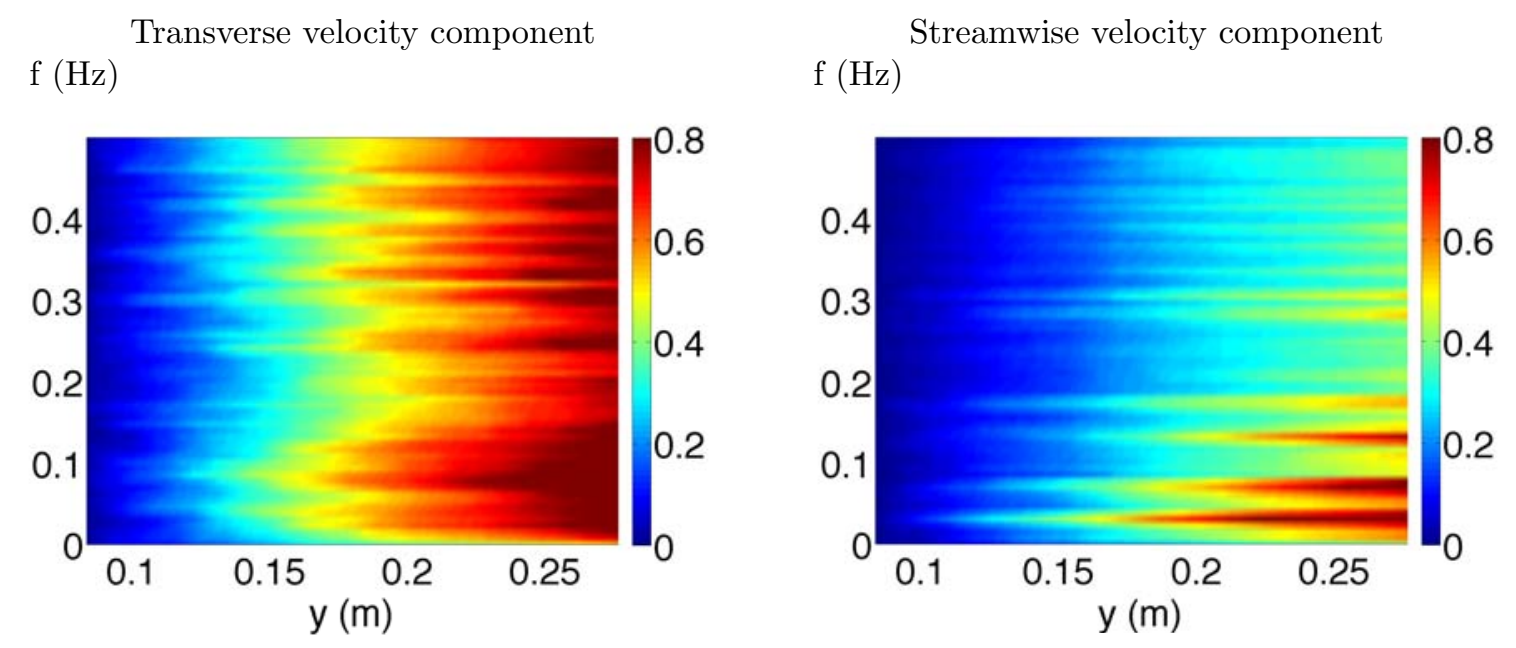

Figure 8: Flow passing through the structure (Plane 11). Normalized frequency spectrum of the transverse (left) and streamwise (right) velocity components along the line $x=2.8 \mathrm{~m}$ and $y>0.05 \mathrm{~m}$.

$\mathrm{y}(\mathrm{m})$
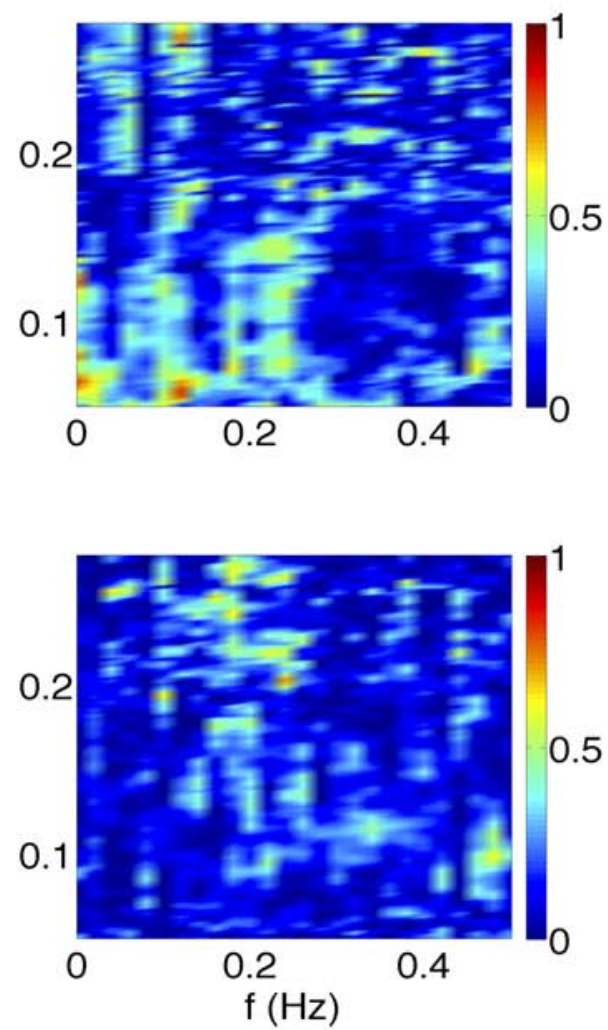

y $(\mathrm{m})$
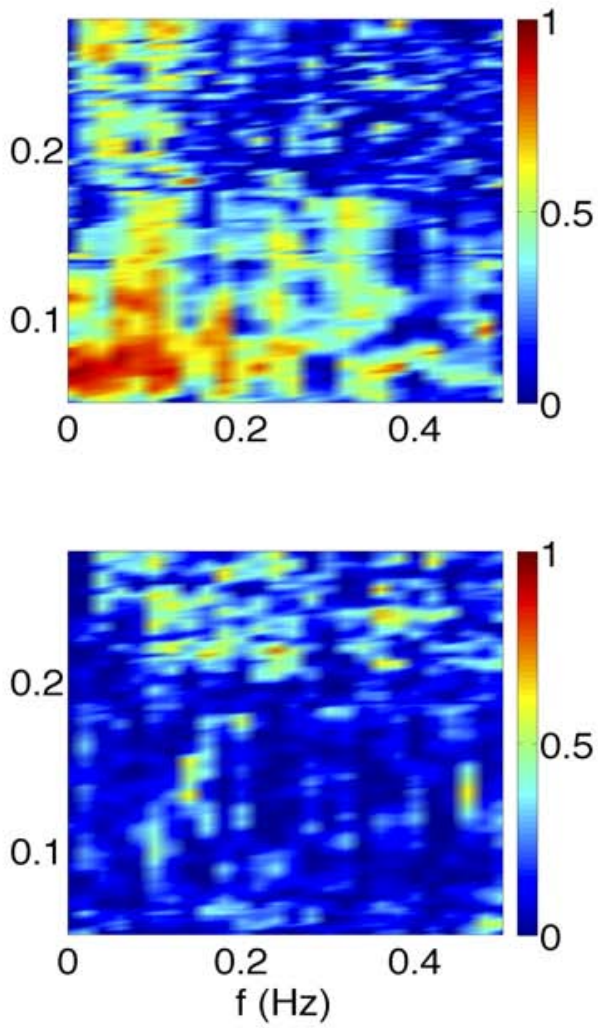

Figure 9: Plane 7. Coherence function computed between streamwise velocity component and $x$-oscillation (top-left) between streamwise velocity component and $y$-oscillation (top-right) between transverse velocity component and $x$-oscillation (bottom-left) between transverse velocity component and $y$-oscillation (bottom-right). 
$\mathrm{y}(\mathrm{m})$
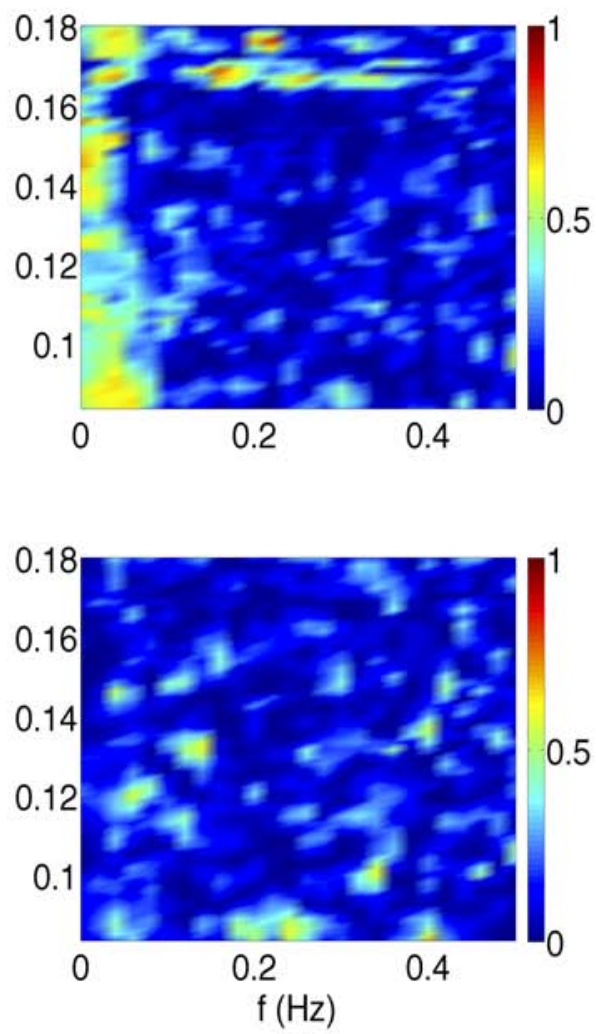

$\mathrm{y}(\mathrm{m})$
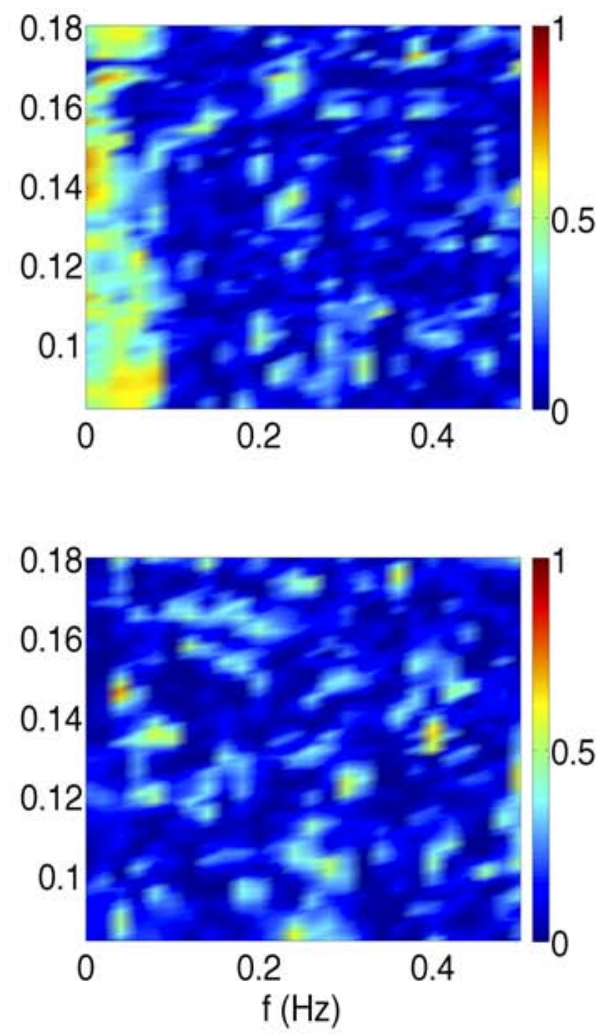

Figure 10: Plane 11. Coherence function computed between streamwise velocity component and $x$-oscillation (top-left) between streamwise velocity component and $y$-oscillation (top-right) between transverse velocity component and $x$-oscillation (bottom-left) between transverse velocity component and $y$-oscillation (bottom-right). 
$y(\mathrm{~m})$

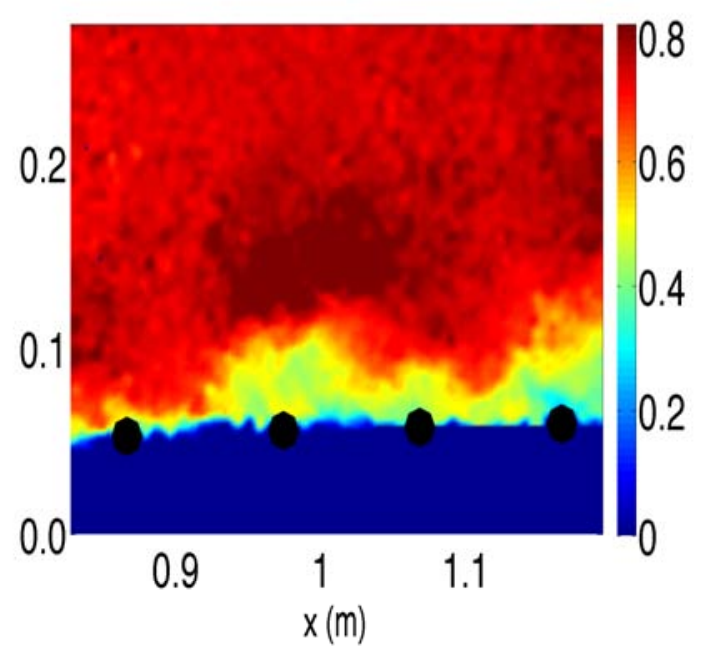

$y(\mathrm{~m})$

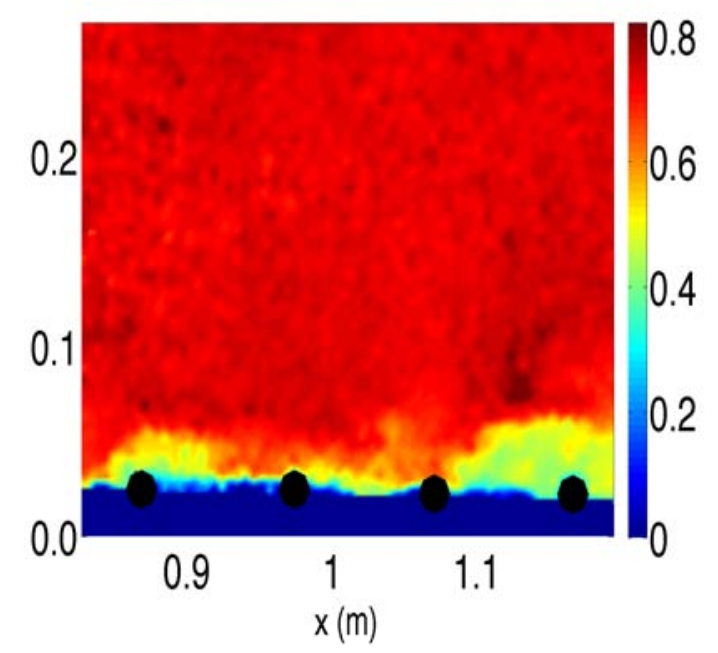

Figure 11: Instantaneous streamwise velocity component obtained at two different instants. Left hand side: when the interface position reaches the crest (highest amplitude). Right hand side: when the interface position reaches its lowest amplitude. Black circles indicate the location of target balls.

\subsubsection{Mean flow field investigation}

As a first illustration, selected instantaneous streamwise velocity components obtained in measurement planes 4 and 7 are displayed in figures 11 and 12 respectively. In figure 11, two velocity fields obtained at two instants related to different positions (crest-highest and lowest amplitudes, respectively) of the porous structure are represented. In figure 12, both retained instants correspond to a quasi-similar position of the porous structure but during an upward and a downward structure motion respectively. The effect of structure motion on the instantaneous streamwise velocity component is then clearly indicated and confirms previous analyses [2]. In each figure, the targets are represented as a black circle and in front of the targets, no available PIV vector fields are obtained.

The time averaged TBL mean flow field is now computed within the structure reference frame which is possible because the motion of the porous structure is instantaneously measured. Applying the tracking system to raw PIV images, the $(x, y)$ coordinates of the structure interface are available in each PIV frame. Then, in each PIV vector velocity field, we re-set at zero the instantaneous velocity vectors in the PIV field located under the detected interface. At each instant (each PIV image) the moving interface is supposed to correspond to the transverse $y$-axis origin. Finally, in each measurement plane, 818 instantaneous PIV vector fields are used to compute the mean flow field in the structure reference frame. It is important to remember that the presence of a target induces a lack of instantaneous velocity fields in the related area. Then, in the area corresponding to the target locations, the transverse $y$-axis origin can not be accurately determined. This generates some small local discontinuities in the resulting mean flow field. Figure 13 represents the resulting mean flow field obtained in measurement planes 4 and 7 . These pictures clearly indicate the spatial development of the TBL flow. To confirm this, figure 13 (bottom-left) displays the mean streamwise velocity component computed in the first seven PIV planes (detailed in figure 1). This picture shows the TBL flow development over the porous structure clearly. As stated above, some small local discontinuities are present and are directly related to the presence of target balls preventing any clear determination of the $y$-axis origin associated with the porous structure.

The mean streamwise velocity components extracted at a fixed streamwise position (that corresponds to the $x$-center of each measurement plane) are also represented as a function of $y$. An analysis of the convergence of the mean velocity computation has been performed leading to ensure the statistical convergence of this computation in each measurement plane. In this graph (bottom-right in figure 
$y(\mathrm{~m})$

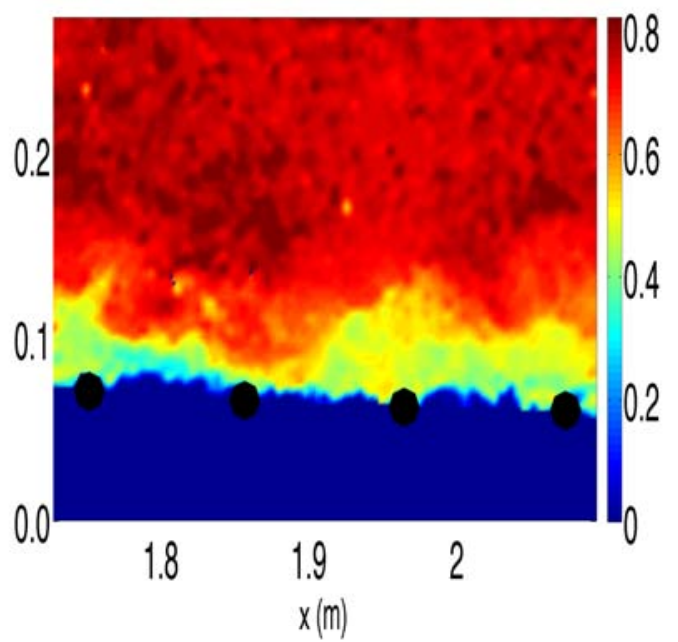

$y(\mathrm{~m})$

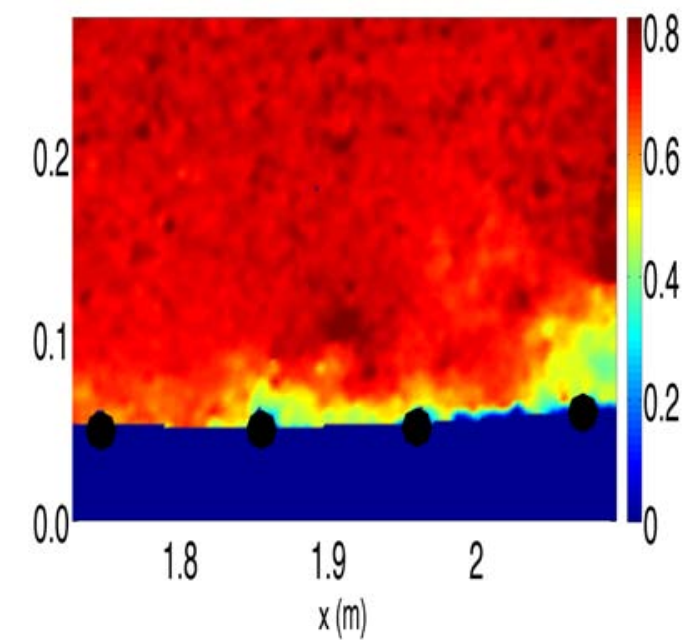

Figure 12: Instantaneous streamwise velocity component obtained at two different instants. Left hand side: when the interface position reaches a mean position but during an upward motion of the structure. Right hand side: when the interface position reaches a mean position but during an downward motion of the structure. Black circles indicate the location of target balls.

13), these mean velocity profiles exhibit different features as a function of streamwise location. In the upstream planes (superior to 3), one observes a rapid increase of the mean flow amplitude in the close vicinity of the net structure. That can be attributed to the local motion of the trawl, which carries out the flow field. Similar behaviours to those previously observed [2] are then recovered.

\subsubsection{Boundary layer thickness investigation}

Based on the available mean flow field, the following usual boundary layer thicknesses [23] are determined: $\delta_{90}$ (and $\delta_{95}$ ) obtained when the mean velocity is equal to $0.9 U_{\text {ref }}$ (and $0.95 U_{\text {ref }}$ ), the displacement thickness $\delta^{*}$ and the momentum thickness $\theta$. They are defined as follows:

$$
\begin{gathered}
\delta^{*}(x)=\int_{0}^{\infty}\left(1-\frac{\overline{u(x, y)}}{U_{\infty}}\right) d y \\
\theta(x)=\int_{0}^{\infty} \frac{\overline{u(x, y)}}{U_{\infty}}\left(1-\frac{\overline{u(x, y)}}{U_{\infty}}\right) d y
\end{gathered}
$$

where $\overline{u(x, y)}$ corresponds to the mean streamwise velocity field. Figure 14 (right hand side) presents the streamwise evolution of these BL thicknesses. They are computed from each mean flow field obtained in each PIV plane assessed. The streamwise evolutions of the TBL thicknesses are quite similar to previous ones computed from TBL flow developing over a horizontal part of a 1/10 scaled fishing trawl [2]. More precisely, Druault et al. [2] found a value $\approx 7.5 \mathrm{~cm}$ for $\delta_{90}$ at a distance of $1 \mathrm{~m}$ from the $x$-beginning of the TBL flow. In the present work, a slightly inferior value is obtained $(5 \mathrm{~cm})$. These differences can be explained firstly by the input streamwise velocity which was twice inferior to the current one and secondly by the small differences related to the determining of the $x$-origin of TBL flows in both studies. 
$y(\mathrm{~m})$

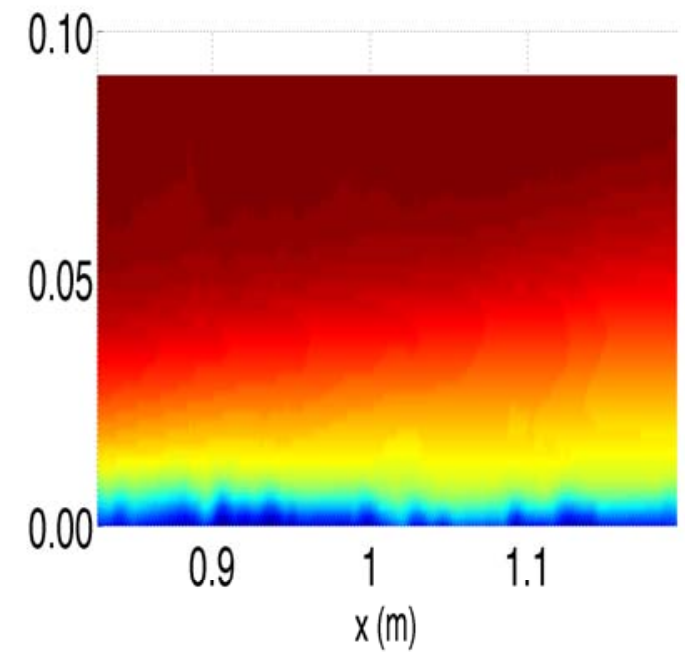

$y(\mathrm{~m}) \quad$ All planes $y(\mathrm{~m})$

Plane 7
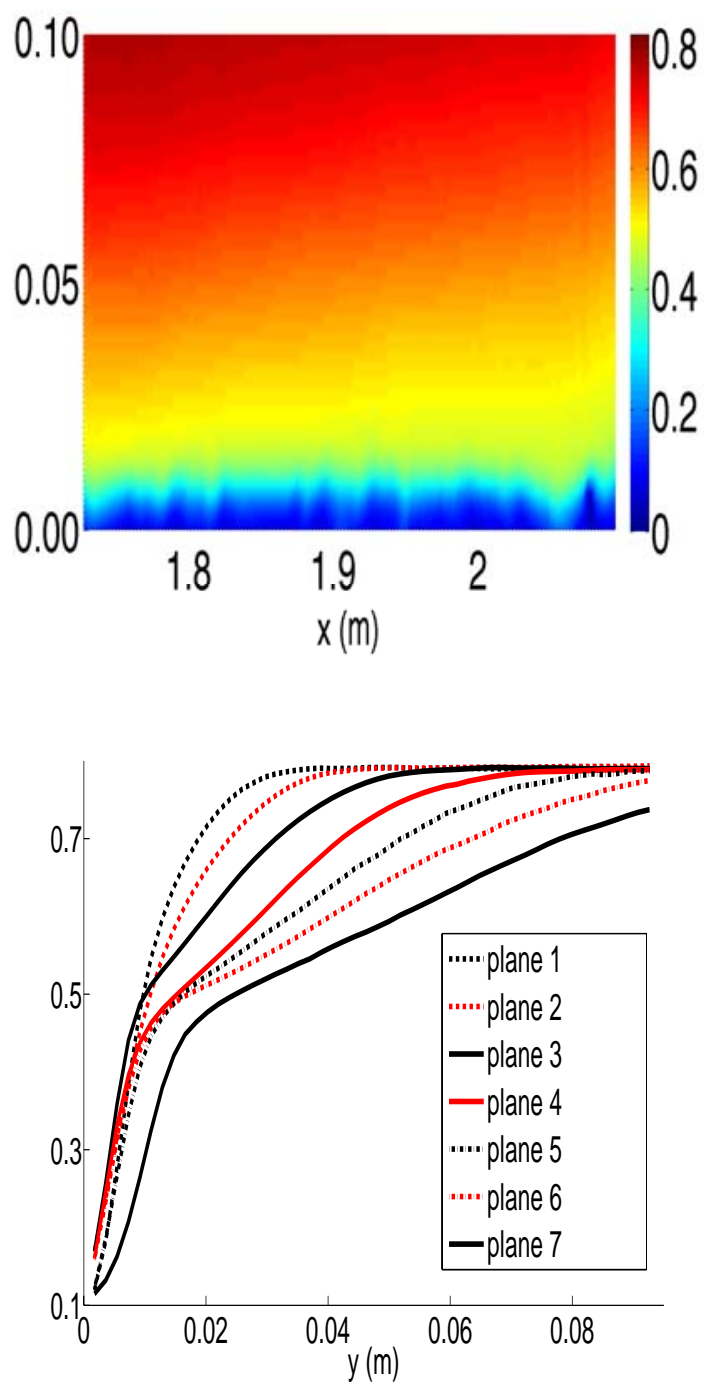

Figure 13: Mean streamwise velocity field computed in the porous structure reference frame. TopLeft: Measurement plane 4. Top-Right: Measurement plane 7. Bottom-Left: Measurement planes from 1 to 7. Bottom-Right: Evolution of the mean streamwise velocity as a function of $y$ extracted at a fixed $x$-position in each measurement plane. 
(m)

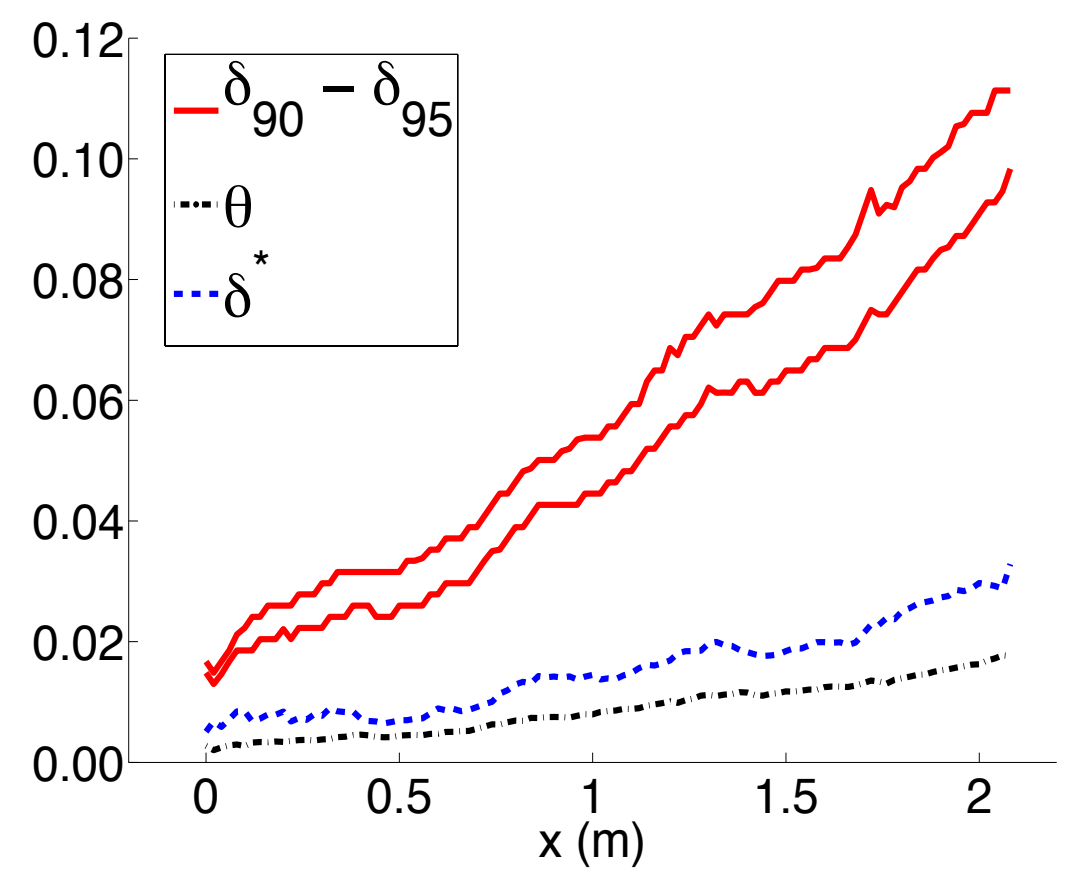

Figure 14: Streamwise evolution of boundary layer thicknesses.

\subsection{Effect of moving porous structure onto the volumetric flow rate}

An investigation of the turbulent flow passing through the inclined moving structure is now presented by observing the volumetric flow rate (along a line segment) as a function of body oscillations. As only $2 \mathrm{D}$ database is available, the volumetric flow rate will only be computed along a vertical line segment in the following. PIV measurements obtained in planes 10 and 11 are considered. The linear flow rate is computed along one vertical line segment in each plane as represented by black vertical lines in figure 1-right hand side. Both vertical line segments are of same lengths, $11.9 \mathrm{~cm}$. In these measurement planes, the body $x$-oscillations are quite negligible compared to the transverse ones (see figure 3). It is therefore only worth comparing the effect of body $y$-oscillations onto the linear flow rate.

Figure 15 displays the time evolution of the linear flow rate along a vertical line segment at a specific $x$-location. It is superimposed onto the time evolution of the transverse $y$-oscillations of the porous structure. Flow rate values are directly linked to the surface (here a segment) scanned size. We then observe that the flow rate values are higher in plane 11 compared to plane 10 . This is directly linked to the incidence of the porous structure and the mesh opening in PIV measurement planes respectively. Based on the fluid velocity measurement around the oscillating structure, we confirm that the incidence has a non-negligible influence on the fluid passing through the porous structure as was previously observed when regarding flow passing sea fish cage [14]. This has a great impact on flux exchange modifying the biological environment of fish and their potential escape [13].

The correlation coefficient between the linear flow rate and the $y$-oscillation signal is computed in each plane. According to planes 10 and 11 results, this coefficient is equal to 0.56 and 0.52 respectively, indicating the existence of a correlation between both signals. This is in agreement with previous results presented in figure 10 where a good coherence has been observed between the streamwise velocity component and $y$-oscillations, especially at low frequency. The low frequency component is indeed the dominant contribution of available signals. In this sense, to emphasize the 

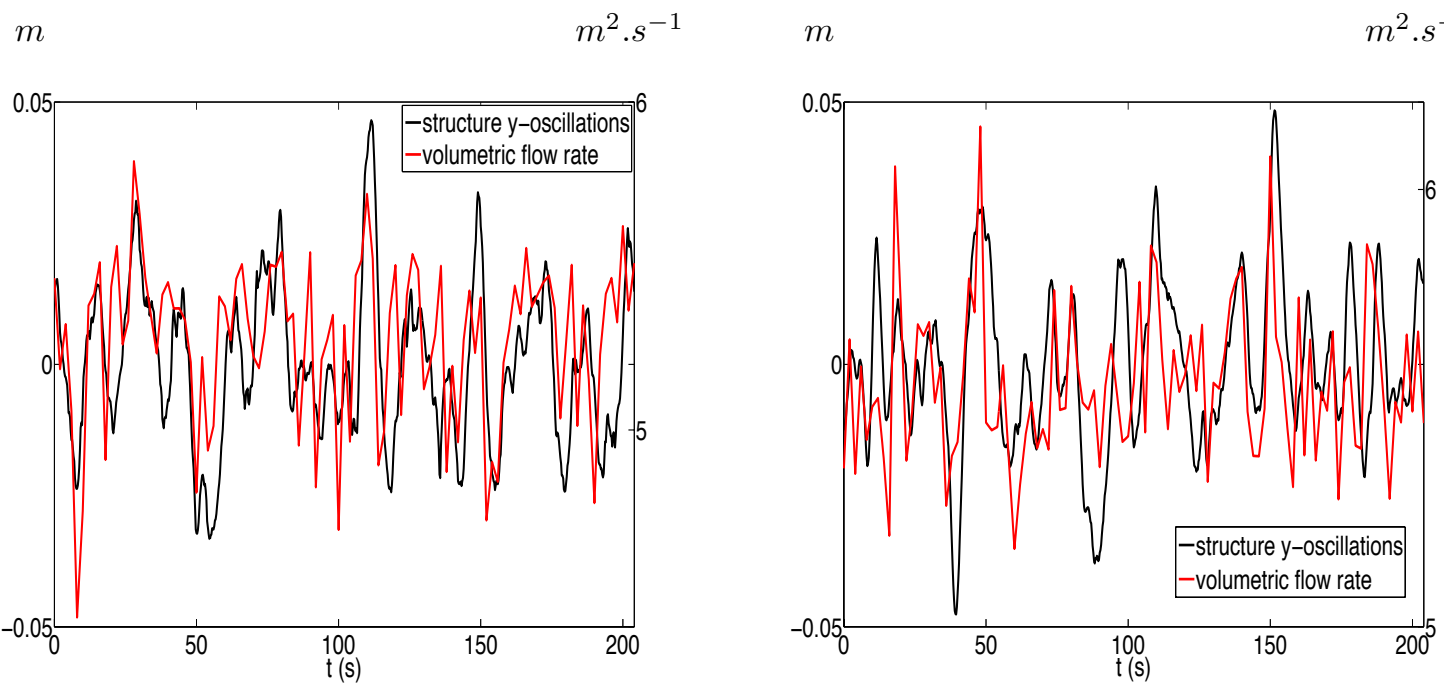

Figure 15: Time evolution of instantaneous linear volumetric flow rate computed along a transverse line segment at a specific $x$-location and superimposed onto the time evolution of the porous structure $y$-oscillations. In each plot, $y$-axes are related to the $y$-oscillations (left $y$-axis) and to the linear flow rate (right $y$-axis). Left hand side: Measurement Plane 10, $x=2.7 \mathrm{~m}$ and $y \in[-21:-9] \mathrm{cm}$. Right hand side: Measurement Plane 11, $x=3 \mathrm{~m}$ and $y \in[-41:-29] \mathrm{cm}$.

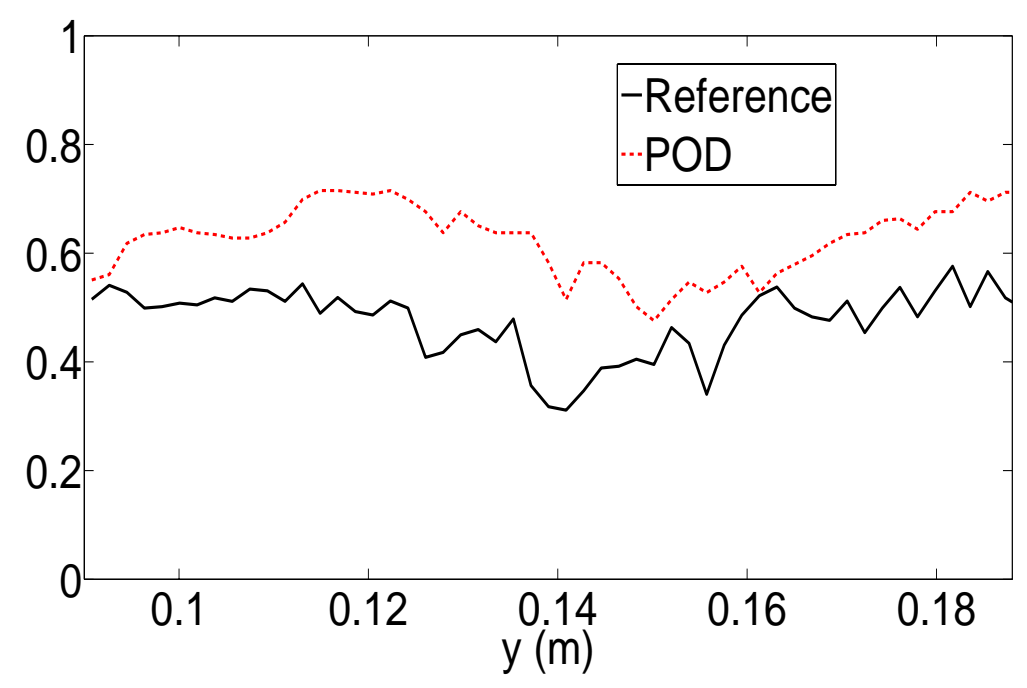

Figure 16: Measurement Plane 10. Correlation between streamwise velocity and $y$-oscillations. 


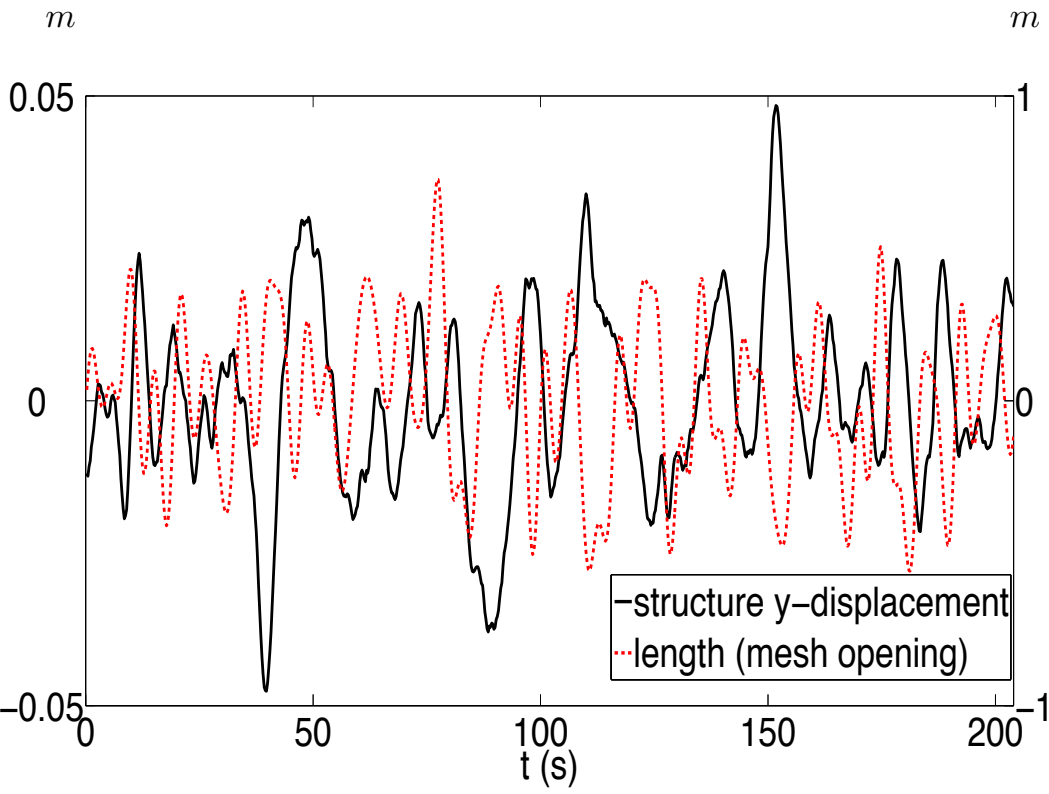

Figure 17: Measurement Plane 11. Time evolution of the fluctuating part of the length between two consecutive balls (dashed line, right $y$-axis) and of the fluctuating part of the $y$-displacement of the structure (black line, left $y$-axis).

relationship between flow rate and $y$-displacement signal, the correlation coefficient between each streamwise velocity stored at each position along the $y$ line and $y$-oscillation signal is computed (see figure 16). Moreover, to limit the effect of background incoherent noise present in velocity signal, one proposes to filter velocity signals. For such an investigation, the procedure given in [19] and based on Proper Orthogonal Decomposition is followed. Briefly, it consists in applying POD to PIV database and then in projecting instantaneous streamwise velocity field onto the first two POD eigenfunctions. Resulted velocity field corresponds then to the phase average mean flow field [19]. The correlation coefficient between $y$-oscillations and the resulted velocity field is also computed. This last correlation coefficient is denoted as $P O D$ in figure 16. A quite good correlation is then obtained especially when dealing with instantaneous projected onto the first two POD modes. The spatial average of the mean correlation coefficient give 0.49 and 0.63 respectively. It is confirmed that the linear flow rate (that is the streamwise flow) computed near the net structure is well correlated with the structure $y$-displacement. More precisely, when the porous structure goes upward, the flow rate increases and inversely. To explain such a result, the net porosity is analyzed thanks to the mesh opening characterization. The net porosity can not be properly investigated in the present study thanks to the available 2D PIV database. Indeed, the porosity is generally defined thanks to the knowledge of the variation of the mesh opening area [24]. Thus, a measure of the length of two consecutive balls located on the fishing net structure allows a first approximation of the mesh opening area (that can be related to mesh porosity). Indeed, as the fishing structure is composed of diamond-shape meshes, when the length of two consecutive balls increases, the mesh opening along a vertical segment is reduced and inversely. The locations of the balls are instantaneously available thanks to the motion tracking system. Then one proposes to examine the time evolution of the length between two consecutive balls. As an illustration, figure 17 displays the time evolution of this length superimposed onto the time evolution of the fluctuating $y$ displacement of the structure. It is then observed that globally both time series are in phase opposition. When the fishing structure goes upward, the length between two consecutive balls decreases and inversely. That confirms previous statement about the influence of fishing net motion on the flow rate. To the author knowledge, it is then the first time that the link between the motion of the net and the mesh opening is clearly 
demonstrated. This link is then explained by two mechanisms: i) the opening of the mesh which increases when the $y$-motion goes upward, and ii) when the structure motion goes downward, the flow passing through the structure follows the motion and a non-negligible transverse flow is observed limiting the volumetric flow rate. It is interesting to observe this phenomenon all around this kind of structure.

\section{Conclusion}

In this work, the TRPIV measurement technique applied to turbulent flow developing over a porous moving deformable structure has been implemented and combined with a synchronous measurement of the motion and deformation of the fishing net structure. After analyzing all the $2 \mathrm{D}$ oscillations of the porous structure, the PIV velocity measurements are analyzed in connection with the detected motion. We then observe that the motion acts on the TBL flow and also onto the fluid behavior through the porous structure.

Working in the reference frame of the porous structure, the mean TBL velocity field is accessed enabling the characterization of the streamwise evolution of the TBL thicknesses. Such a description of the flow reinforces the fact that locally a deficit of velocity is observed specifically along the horizontal part of fishing gears. As stated in the introduction, numerical code aimed at computing the fishing gear drag force is somewhat limited due to the lack of available velocity database. Thus, instead of using a constant velocity field in our numerical model, it will be possible to use empirical formulations taking into account the local deficit of velocity along the horizontal parts of a fishing gear [25]. Such new formulations will probably improve the computation of the whole drag force.

Moreover, the effect of porous structure oscillations in the volumetric flow rate has been clearly demonstrated. Indeed, we observe that the turbulent flow passes favorably through the porous structure especially when the fishing net structure moves in an upward motion. This motion leads to cyclic changes in the mesh opening which may increase the probability of undersized fish escaping. Such a result may also have implications in the aquaculture fish cage where the flow reduction passing through the net panel modifies for example the oxygen distribution and then the biological environment of fish [13]. It would be interesting in the future to investigate the effect of incidence and structure oscillation on the flow passing fish cage and then on the fish behavior and physiology.

As it has been previously observed, cod-end pulsing (and associated cod-end hydrodynamics) influence especially the selectivity or the reduction of by-catch of the cod-end [26]. Indeed, we observe that not only the longitudinal pulsing of the cod-end seems to be the dominant but also the vertical oscillations. This study has confirmed that transverse oscillations of the fishing structure induce mesh opening variabilities which may have a significant influence on the escape of fish. Furthermore, as noticed before [21], the turbulent flow inducing the fluttering process acts as an active stimulating device which increases the possibility of fish escaping. In the near future, it will be interesting to pursue the current analysis to investigate the turbulent flow and also the tilt motion and its influence on fish swimming speed and postural control as in [27]. A particularly useful future investigation concerns the characterization of the turbulent boundary layer which develops under the moving porous structure. Such a characterization will enable us to fully understand how juvenile fish may attempt to approach the netting and may escape from the cod-end.

\section{Acknowledgements}

The authors would like to gratefully acknowledge E. Bouhoubeiny and B. Gaurier for their involvement in the experimental work. We also acknowledge the French Ministry of Agriculture and Fisheries and the European Community (Convention number 31218/2009) for their financial support during the HydroPêche project monitored by IFREMER (Institut Français de Recherche pour l'Exploitation de la MER). 


\section{References}

[1] Y. Jeon, H. Sung, Piv measurement of flow around an arbitrarily moving body, Exp. Fluids 50 (2011) 787-798.

[2] P. Druault, E. Bouhoubeiny, G. Germain, POD investigation of the unsteady turbulent boundary layer developing over porous moving flexible fishing net structure, Exp. Fluids 53 (1) (2012) $277-292$.

[3] D. Khalitov, E. Longmire, Simultaneous two-phase PIV by two-parameter phase discrimination, Exp. Fluids 32 (2002) 252-268.

[4] D. Adhikari, E. Longmire, Visual hull method for tomographic PIV measurements of flow around moving objects, Exp. Fluids 53 (4) (2012) 943-964.

[5] E. Bouhoubeiny, G. Germain, P. Druault, Time-resolved PIV investigations of the flow field around rigid cod-end net structure, Fisheries Research 108 (2-3) (2011) 344-355.

[6] D. Priour, Numerical optimisation of trawls design to improve their energy efficiency, Fisheries Research 98 (2009) 40-50.

[7] Z. Zheng, N. Zhang, Frequency effects on lift and drag for flow past an oscillating cylinder, J. Fluids Struct. 24 (2008) 382-399.

[8] L. Liu, T. Kinoshita, R. Wan, W. Bao, H. Itakura, Experimental investigation and analysis of hydrodynamic characteristics of a net panel oscillating in water, Ocean Eng. 47 (2012) 19-29.

[9] G. Pichot, G. Germain, D. Priour, On the experimental study of the flow around a fishing net, Eur. J. Mech. B/Fluids (2009) 103-116.

[10] M. Paschen, H. Winkel, H. Knuths, Fluid-structure interactions in pelagic trawls and probable consequences for the selectivity of the fishing gear, Adv. Science Techn. 58 (2008) 247-256.

[11] Y. Kim, Analysis of turbulence and tilt by in-situ measurements inside the cod-end of a shrimp beam trawl, Ocean Eng. 53 (2012) 6-15.

[12] P. Lader, T. Dempster, A. Fredheim, O. Jensen, Current induced net deformation in full-scale sea cages for atlantic salmon (salmo solar), Aqua. Eng. 38 (2008) 52-65.

[13] P. Klebert, P. Lader, L. Gansel, F. Oppedal, Hydrodynamic interactions on net panel and aquaculture fish cages: A review, Ocean Eng. 58 (2013) 260-274.

[14] L. Gansel, T. McClimans, D. Myrhaug, Flow around the free bottom of fish cages in a uniform flow with and without fouling, J. Offshore Mech. Arct. Eng. 134 (2011) 011501.1-8.

[15] C. Tomkins, R. Adrian, Spanwise structure and scale growth in turbulent boundary layers, J. Fluid Mech. 490 (2003) 37-74.

[16] A. vision. MA STUDIO 3.2.1, Ma-Studio (Motion Analysis software), www.alliancevision.eu/products/softwares-drivers/image-processing-and-analysissoftwares/ma-studio-motion-analysis-software/.

[17] Rajogopal, R., Pattern matching based on a generalized transform., Tech. rep., National Instruments (Austin, Texas) (2000).

[18] E. Bouhoubeiny, Caractérisation de l'écoulement autour de structures souples et poreuses : Application aux engins de pêche, Ph.D. thesis, University Pierre and Marie Curie - Paris 6 (2012). 
[19] E. Bouhoubeiny, P. Druault, G. Germain, Phase-averaged mean properties of turbulent flow developing around a fluttering sheet of net, Ocean Eng. 82 (2014) 160-168.

[20] J. Facq, T. Bacchetti, G. Germain, B. Gaurier, Compte rendu d'essai de mesures d'efforts et de géométries sur une nappe d'alèse (in french), Tech. rep., IFREMER (2010).

[21] Y. Kim, D. Whang, An actively stimulating net panel and rope array inside a model cod-end to increase juvenile red seabream escapement, Fisheries Research 106 (2010) 71-75.

[22] E. Anderson, W. McGillis, M. Grosenbaugh, The boundary layer of swimming fish., J Exp. Biol. 204 (2001) 81-102.

[23] H. Schlichting, K. Gersten, Boundary layer theory. 8th revised and enlarged edition, Springer, 2000.

[24] S. Gjosund, B. Enerhaug, Flow through nets and trawls of low porosity, Ocean Eng. 37 (2010) $345-354$.

[25] I. Mnassri, Modélisation 3D des écoulements guidés par des parois perméables mobiles. Application aux problèmes de technologies halieutiques, Ph.D. thesis, Ecole Centrale Nantes (2012).

[26] F. O'Neill, S. McKay, J. Ward, A. Strickland, R. Kynoch, A. Zuur, An investigation of the relationship between sea state induced vessel motion and cod-end selection, Fisheries Research 60 (2003) 107-130.

[27] H. Tritico, A. Cotel, The effects of turbulent eddies on the stability and critical swimming speed of creek chub, J. Exp. Biol. 213 (2010) 2284-2293. 